\title{
Multi-institutional analysis of T3 subtypes and adjuvant radiotherapy effects in resected T3NO non-small cell lung cancer patients
}

\author{
Yunseon Choi, MD ${ }^{1,2}$, Ik Jae Lee, MD¹, Chang Young Lee, MD³, Jae Ho Cho, MD', Won Hoon Choi, PhD', \\ Hong In Yoon, MD', Yun-Han Lee, PhD', Chang Geol Lee, MD¹, Ki Chang Keum, MD', \\ Kyung Young Chung, MD³, Seok Jin Haam, MD³, Hyo Chae Paik, MD³, Kang Kyoo Lee, MD,

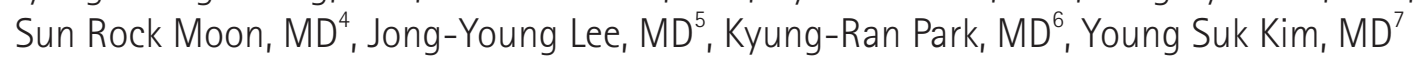 \\ 'Department of Radiation Oncology, Severance Hospital, Yonsei University College of Medicine, Seoul; \\ ${ }^{2}$ Department of Radiation Oncology, Busan Paik Hospital, Inje University College of Medicine, Busan; \\ ${ }^{3}$ Department of Thoracic and Cardiovascular Surgery, Severance Hospital, Yonsei University School of Medicine, Seoul; \\ ${ }^{4}$ Department of Radiation Oncology, Wonkwang University School of Medicine, Iksan; \\ ${ }^{5}$ Department of Radiation Oncology, Wonju Severance Christian Hospital, Wonju; \\ ${ }^{6}$ Department of Radiation Oncology, Ewha Womans University Hospital, Seoul; \\ ${ }^{7}$ Department of Radiation Oncology, Jeju National University Hospital, Jeju University College of Medicine, Jeju, Korea
}

Purpose: We evaluated the prognostic significance of T3 subtypes and the role of adjuvant radiotherapy in patients with resected the American Joint Committee on Cancer stage IIB T3NOMO non-small cell lung cancer (NSCLC).

Materials and Methods: T3NO NSCLC patients who underwent resection from January 1990 to October $2009(n=102)$ were enrolled and categorized into 6 subgroups according to the extent of invasion: parietal pleura chest wall invasion, mediastinal pleural invasion, diaphragm invasion, separated tumor nodules in the same lobe, endobronchial tumor $<2 \mathrm{~cm}$ distal to the carina, and tumor-associated collapse.

Results: The median overall survival (OS) and disease-free survival (DFS) were 55.3 months and 51.2 months, respectively. In postoperative T3NOMO patients, the tumor size was a significant prognostic factor for survival (OS, $p=0.035$ and DFS, $p=0.035$, respectively). Patients with endobronchial tumors within $2 \mathrm{~cm}$ of the carina also showed better OS and DFS than those in the other T3 subtypes ( $p=0.018$ and $p=0.016$, respectively). However, adjuvant radiotherapy did not cause any improvement in survival (OS, $p=0.518$ and DFS, $p=0.463$, respectively). Only patients with mediastinal pleural invasion $(n=25)$ demonstrated improved OS and DFS after adjuvant radiotherapy $(n=18)(p=0.012$ and $p=0.040$, respectively).

Conclusion: The T3NO NSCLC subtype that showed the most favorable prognosis is the one with endobronchial tumors within 2 $\mathrm{cm}$ of the carina. Adjuvant radiotherapy is not effective in improving survival outcome in resected T3NO NSCLC.

Keywords: Non-small cell lung cancer, Adjuvant radiotherapy, Prognostic factor

Received 26 January 2015, Revised 13 March 2015, Accepted 20 March 2015.

Correspondence: Ik Jae Lee, MD, Department of Radiation Oncology, Gangnam Severance Hospital, Yonsei University College of Medicine, 211 Eonju-ro, Gangnam-gu, Seoul 135-720, Korea. Tel: +82-2-2019-3154, Fax: +82-2-2019-4821, E-mail: ikjae412@yuhs.ac

(c) This is an Open Access article distributed under the terms of the Creative Commons Attribution Non-Commercial License (http://creativecommons.org/ licenses/by-nc/4.0/) which permits unrestricted non-commercial use, distribution, and reproduction in any medium, provided the original work is properly cited.

www.e-roj.org 


\section{Introduction}

The role of adjuvant therapy in patients with resected nonsmall cell lung cancer (NSCLC) has been evaluated in many randomized clinical trials and meta-analyses [1-3]. Although complete resection has been consistently considered to be the treatment of choice for most NSCLC patients with T3 disease, including cases with chest wall invasion, the role of adjuvant treatment following complete surgical resection of early-stage NSCLC has not been fully established [4-6]. T3NO NSCLC is a heterogeneous group of tumor subtypes. According to the 6th edition of the American Joint Committee on Cancer (AJCC) staging system, patients with pT3NO tumors show a better 5 -year survival rate than those with pT2NO tumors $>7 \mathrm{~cm}(41 \%$ vs. 35\%, respectively), while in patients with T4 tumors with additional nodules in the same lobe the 5 -year overall survival (OS) rate was 31\% [7]. In the 7th edition of the AJCC, T3 stage includes various subtypes, particularly tumors that measure $>7 \mathrm{~cm}$ and those where at least two tumors are existent in the same lobe. These two subtypes have been newly included in 2010 in the 7th edition of the cancer staging manual of AJCC [8]. Surgery is considered the treatment of choice in patients with T3NO NSCLC, and surgical technique has improved with time [9]. The survival of pT3 NSCLC patients changes according to the completeness of the resection, and a previous study reported that the prognosis of patients with completely resected tumors was not affected by the depth of invasion, even in the presence of rib or parietal pleura invasion [10].

Controversy still exists concerning whether adjuvant radiotherapy should be performed for patients with postoperative stage T3NO cancer [2]. Moreover, various opinions have been proposed regarding whether the radiation field should be limited to the region of the primary tumor or extended to the mediastinal Iymph node area $[4,5]$. A Surveillance, Epidemiology and End Results (SEER) database analysis reported that postoperative radiotherapy was efficacious for patients with stage N2 cancer but lowers the survival rate of patients with stage N0 and N1 cancer $[11,12]$. However, one of the critical limitations of the previous study, due to its retrospective and unmatched design, was that the patients who underwent postoperative radiotherapy had more risk factors for recurrence $[13,14]$. Additionally, it has been pointed out that some patients might have been subjected to radiotherapy with an inappropriate dose or field $[11,15]$.

Here, we evaluated the prognostic significance of T3 subtypes and the role of postoperative radiation therapy in resected stage IIB T3NOMO NSCLC patients. The study aimed to evaluate the prognostic factors for patients who underwent resection for stage IIB T3NOMO NSCLC. First, the prognosis of patients was evaluated according to T3 subtype. In addition, we focused on evaluating the efficacy of adjuvant radiotherapy. Using subgroup analyses, we tried to identify a subgroup of patients who might benefit from adjuvant radiotherapy.

\section{Materials and Methods}

\section{Patients}

In the present study, we conducted a multi-institutional retrospective analysis of 102 patients with postoperative stage T3NO NSCLC at Wonkwang University Hospital, Wonju Severance Christian Hospital, Gangnam Severance Hospital, and Shinchon Severance Hospital. The patients had undergone resection from January 1990 until October 2009 and were selected in accordance with the 7th edition AJCC stage T3NO. Before undergoing the operation, each patient underwent staging workups such as chest computed tomography (CT) and whole-body bone scans. Because the descriptions of T3 tumor extent vary, we categorized the patients into 6 subgroups for T3 tumors: parietal pleura chest wall invasion, mediastinal pleural invasion, diaphragm invasion, separated tumor nodules in the same lobe, endobronchial tumor located $<2 \mathrm{~cm}$ distal to the carina, tumor-associated collapse (atelectasis) or obstructive pneumonia (Fig. 1). This study was approved by our

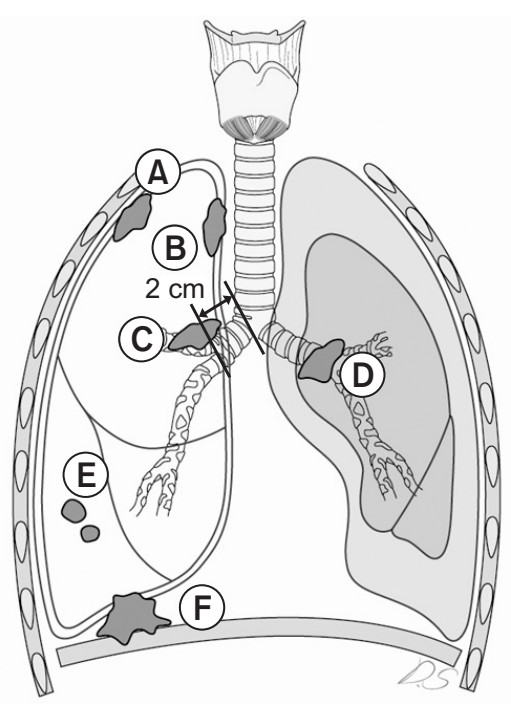

Fig. 1. Schematic illustration of non-small cell lung cancer. (A) Parietal pleura/chest wall invasion. (B) Mediastinal pleural invasion. (C) Endobronchial tumor $<2 \mathrm{~cm}$ distal to the carina. (D) Tumorassociated collapse (atelectasis) or obstructive pneumonia. (E) Separated tumor nodules in the same lobe. (F) Diaphragm invasion. 
Institutional Review Board (IRB No. 3-2011-0164). The median follow-up period was 35.9 months.

\section{Treatment}

Complete resections were performed in all but 5 patients (95.1\%). When chest wall invasion was preoperatively suspected, a chest wall resection, including the affected rib, was scheduled. Extrapleural resection was performed when the parietal pleura could be easily freed from the chest wall. Five patients (4.9\%) underwent a complete gross resection by thoracotomy but were found to have positive margins on the final pathologic review of the resected specimens ( $R 1$ resections). Seventy-six patients (74.5\%) manifested pleural invasion (parietal pleura/chest wall invasion + mediastinal pleural invasion), while 16 (15.7\%) were diagnosed with an endobronchial lesion within $2 \mathrm{~cm}$ of the carina (Table 1). Adjuvant radiotherapy was administered at 6 or $10 \mathrm{MV}$ photons using a linear accelerator in 57 patients (55.9\%). The median total radiation dose was $54 \mathrm{~Gy}$ (range, 45 to $60 \mathrm{~Gy}$ ).

Table 1. Patient characteristics $(n=102)$

\begin{tabular}{lc}
\hline \multicolumn{1}{c}{ Characteristic } & No. (\%) \\
\hline Sex (male) & $85(83.3)$ \\
Tumor size $(\geq 5 \mathrm{~cm})$ & $56(54.9)$ \\
Tumor location & \\
Left upper lobe & $34(33.3)$ \\
Right upper lobe & $35(34.3)$ \\
Right middle lobe & $5(4.9)$ \\
Right lower lobe & $13(12.7)$ \\
Left lower lobe & $13(12.7)$ \\
Right main bronchus & $1(1.0)$ \\
Left main bronchus & $1(1.0)$ \\
T3 subtype & \\
Parietal pleura/chest wall invasion & $51(50.0)$ \\
Mediastinal pleural invasion & $25(24.5)$ \\
Same lobe multiple lesions & $4(3.9)$ \\
Endobronchial lesion, carina $<2$ cm & $16(15.7)$ \\
Collapse with obstruction & $3(2.9)$ \\
Diaphragm invasion & $3(2.9)$ \\
Risk factor & \\
Lymphovascular invasion & $6(5.9)$ \\
Vascular invasion & $7(6.9)$ \\
Resection margin positive & $5(4.9)$ \\
Histological feature & \\
Squamous cell carcinoma & $5(2.0)$ \\
Adenocarcinoma & $6(5.9)$ \\
Adenosquamous carcinoma & \\
Large cell carcinoma & \\
Non-small cell lung cancer, others & \\
\hline & \\
\hline
\end{tabular}

The radiation field included the tumor bed without/with the mediastinal lymph node areas. The tumor bed was defined as the region invaded by the tumor and the surrounding tissue (primary tumor bed plus the margin). Platinum-based CT agents (docetaxel or paclitaxel + cisplatin) were mainly used as an adjuvant treatment.

\section{Follow-up and response assessment}

The OS was measured between the date of operation and the date of death or final observation, and disease-free survival (DFS) was measured between the date of operation and the date of recurrence. The locoregional relapse-free survival and distant metastasis-free survival were also evaluated. Local failure was defined as tumor recurrence within the original tumor bed. Regional failure was defined as tumor recurrence within the ipsilateral hilar, mediastinal, or supraclavicular regions. Distant metastasis refers to cancer that has spread from the original (primary) tumor to distant organs or distant lymph nodes.

For statistical analysis, PASW ver. 18 (SPSS Inc., Chicago, IL, USA) was used. The Kaplan-Meier method and the log-rank test were used to calculate the survival rate and to compare the scores of subgroups, respectively. For multivariate analysis, Cox regression was used.

\section{Results}

\section{Patient characteristics}

The median age of the patients was 64 years (range, 37 to 85 years), and 85 patients (83.3\%) were male. The median size of the tumors was $5 \mathrm{~cm}$, and 55 patients (55.6\%) had tumors that measured $5 \mathrm{~cm}$ and above. Sixty-nine patients (67.6\%) had tumors located in the upper lobe. A similar number of tumors were present in the left upper lobe $(n=34)$ and right upper lobe $(n=35)$. The most common T3 subtype was pleural invasion, present in 76 patients (74.5\%), followed by an endobronchial lesion within $2 \mathrm{~cm}$ of the carina in 16 patients (15.7\%). The main histologic types were squamous cell carcinoma (57.8\%) and adenocarcinoma (30.4\%). Of 102 patients, 57 (55.9\%) underwent adjuvant radiotherapy. Among the patients who underwent radiotherapy ( $n=57), 43$ patients (75.4\%) received mediastinal irradiation; eleven of these patients had costal pleural invasion (i.e., elective radiotherapy to mediastinum). Fifty-two patients (51.0\%) received adjuvant chemotherapy (Table 2). 


\section{Survival analysis}

The median OS was 55.3 months and the median DFS was 51.2 months (Fig. 2). The actuarial 4-year loco-regional relapsefree survival was 49.9\%, and the 4-year distant metastasisfree survival was 53\%. The univariate analysis showed that OS $(p=0.008)$ and DFS $(p=0.004)$ were significantly lower in tumors that measured $5 \mathrm{~cm}$ and above (Table 3). Patients with endobronchial tumors located $<2 \mathrm{~cm}$ distal to the carina showed higher survival rate than other T3 subtypes in the univariate analysis $(0 S, p=0.003$ and DFS, $p=0.006$, respectively). Also, the patients with pleural invasion showed lower survival than other T3 subtypes in the univariate analysis (OS, $p=0.023$ and DFS, $p=0.049$, respectively). On multivariate analysis, the OS and DFS were significantly lower in tumors that measured $\geq 5 \mathrm{~cm}$ than in tumors $<5 \mathrm{~cm}$ ( $p$ $=0.035$ and $p=0.035$, respectively) (Table 3 ). The location of endobronchial tumors $<2 \mathrm{~cm}$ distal to the carina was an independent prognostic factor for OS and DFS ( $p=0.018$ and $p=0.016$, respectively) (Table 3 ). The survival rate according to operation type and extent of resection was analyzed, and no statistically significant difference was noted in survival (Tables 3). The OS showed no significant difference between the

Table 2. Treatment characteristics $(n=102)$

\begin{tabular}{lc}
\hline Characteristic & No. (\%) \\
\hline Operation type & \\
Pneumonectomy & $30(29.4)$ \\
Lobectomy & $64(62.7)$ \\
Bilobectomy & $3(2.9)$ \\
Wedge resection & $3(2.9)$ \\
Sleeve lobectomy & $2(2.0)$ \\
Adjuvant radiotherapy & \\
$\quad$ Radiotherapy & $57(55.9)$ \\
No radiotherapy & $45(44.1)$ \\
Adjuvant chemotherapy & \\
Chemotherapy & $52(51.0)$ \\
No chemotherapy & $50(49.0)$ \\
\hline
\end{tabular}

pneumonectomy group $(n=30)$ and the lobectomy $(n=64)$ group ( $p=0.417$ vs. $p=0.601$, respectively). Wedge resection was performed only on 3 patients and showed high treatment failure rates $(66.7 \%)$. The patients who underwent wedge resection showed a lower survival rate (4-year OS, 33.3\% and 4-year DFS, 33.3\%, respectively) than the patients treated with other types of curative resection. These patients underwent wedge resections owing to tuberculosis $(n=2,2.0 \%)$ or emphysema $(n=1,1.0 \%)$ or bronchiectasis $(n=1,1.0 \%)$. No significant difference was found in OS and DFS between patients treated with or without adjuvant chemotherapy $(p=$ 0.440 and $p=0.619$, respectively).

\section{Survival implication of T3 heterogeneity}

The T3 subtype significantly correlated with patient survival in the present study. Table 3 shows significantly low OS and DFS in stage T3NO patients with pleural invasion in univariate analysis ( $p=0.023$ and $p=0.049$, respectively). Patients with endobronchial tumors within $2 \mathrm{~cm}$ of the carina also had better survival than patients with other $\mathrm{T3}$

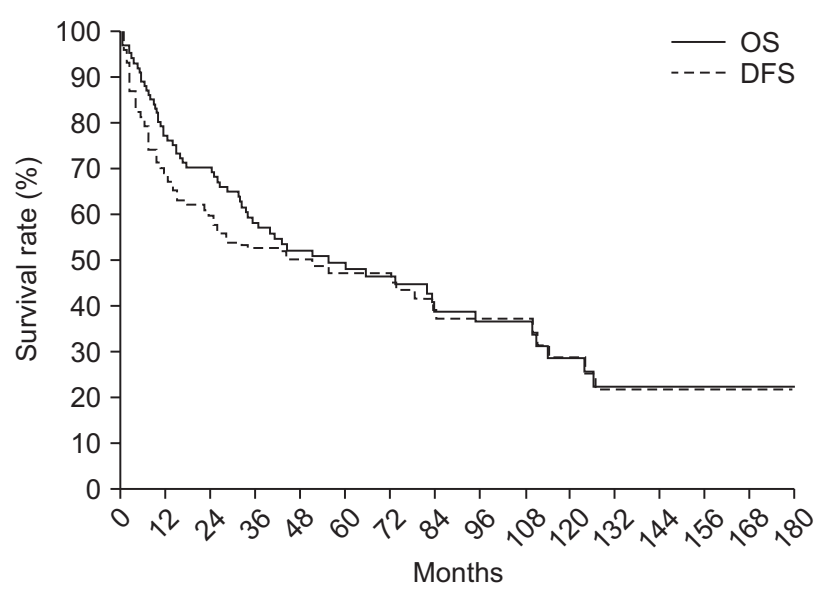

Fig. 2. Overall survival (OS) and disease-free survival (DFS) among the 102 patients. The median OS was 55.3 months and the median DFS was 51.2 months.

Table 3. Univariate analysis (log-rank test) and multivariate analysis (Cox regression) for survival

\begin{tabular}{|c|c|c|c|c|}
\hline \multirow{2}{*}{ Variable } & \multicolumn{2}{|c|}{ Univariate analysis (p-value) } & \multicolumn{2}{|c|}{ Multivariate analysis ( $p$-value) } \\
\hline & Overall survival & Disease-free survival & Overall survival & Disease-free survival \\
\hline Tumor size $(\geq 5 \mathrm{~cm}$ vs. $<5 \mathrm{~cm})$ & 0.008 & 0.004 & 0.035 & 0.035 \\
\hline Endobronchial lesion (carina $<2 \mathrm{~cm}$ ) & 0.003 & 0.006 & 0.018 & 0.016 \\
\hline Pleural invasion & 0.023 & 0.049 & 0.461 & 0.550 \\
\hline Adjuvant chemotherapy & 0.204 & 0.304 & - & - \\
\hline Adjuvant radiotherapy & 0.518 & 0.463 & - & - \\
\hline Operation type & 0.734 & 0.692 & - & - \\
\hline
\end{tabular}


subtypes in univariate analysis and multivariate analysis (Table 3). Regarding the T3 subtype with endobronchial tumors within $2 \mathrm{~cm}$ of the carina, the tumor size was comparatively smaller than that of other T3 subtypes. Though there was no significant correlation in multivariate analysis, most patients with endobronchial tumors within $2 \mathrm{~cm}$ of the carina (10/16, $62.5 \%$ ) presented with a tumor $<5 \mathrm{~cm}$.

\section{The impact of adjuvant radiotherapy on tumor location and radiotherapy}

Overall, adjuvant radiotherapy did not improve patient survival in the present study (Table 3). However, the patients who received adjuvant radiotherapy had more risk factors. Four of 5 patients (80\%) with a positive resection margin and 4 patients

A

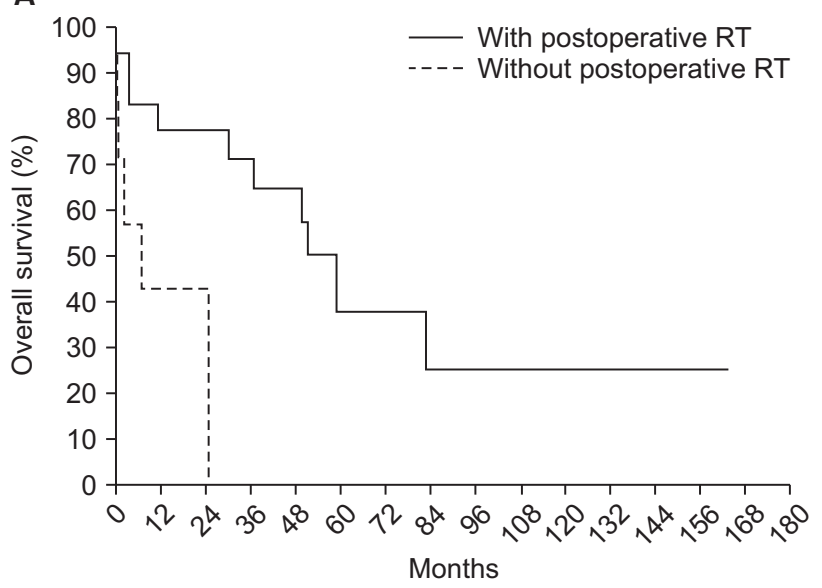

(66.7\%) with lymphovascular invasion received postoperative radiotherapy.

In cases of mediastinal pleural invasion ( $n=25), 0 S$ and DFS were longer in patients treated with postoperative radiotherapy than in patients who did not receive radiotherapy ( $n=18$ ) (OS, $p=0.012$ and DFS, $p=0.040$, respectively) (Fig. 3).

Among the patients who had accompanying costal pleura invasion and received irradiation ( $n=22)$, a subgroup analysis of the radiation field was performed; the survival rate was higher in cases where radiotherapy was administered only to the costal pleura than in cases where radiotherapy was also administered to the mediastinum (OS, $p=0.037$ and DFS, $p=$ 0.009) (Fig. 4). Patients who received radiotherapy only to the costal pleura $(n=11)$ experienced more episodes of recurrence

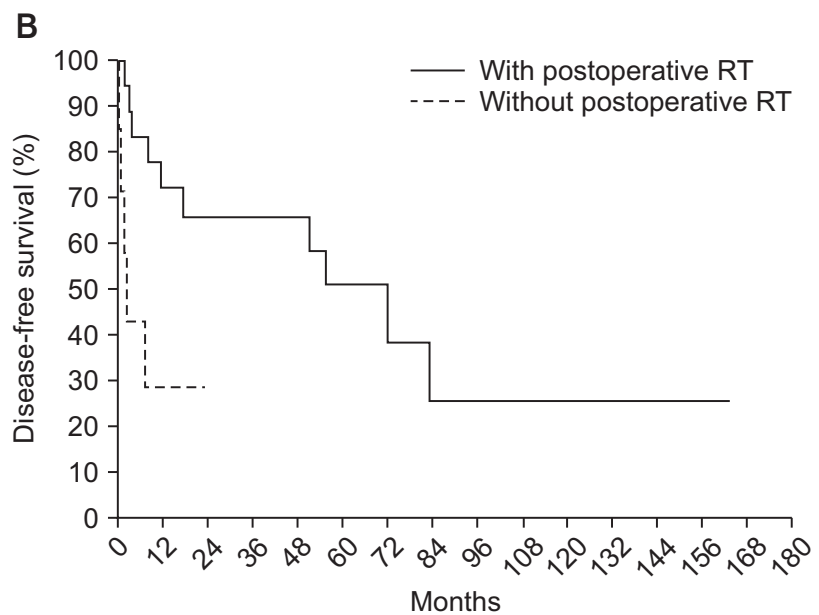

Fig. 3. Postoperative radiotherapy $(n=18)$ improved $(A)$ overall survival $(p=0.012)$ and $(B)$ disease-free survival $(p=0.040)$ in patients with mediastinal pleural invasion $(n=25)$.
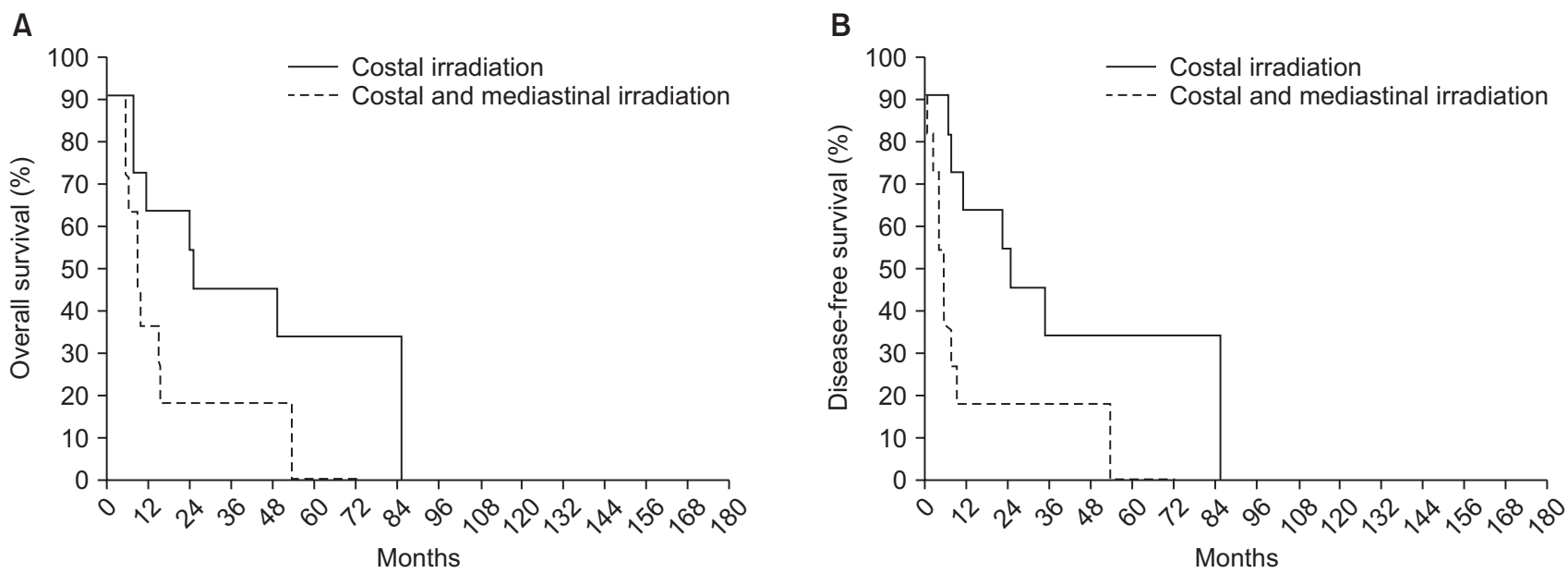

Fig. 4. In patients who underwent postoperative radiotherapy (PORT) for lesions invading the costal pleura ( $n=22)$, ( $\mathrm{A})$ overall survival rate $(p=0.037)$ and $(B)$ disease-free survival $(p=0.009)$ rate were higher in patients who received PORT only to the costal pleura $(n=$ 11) than in those who also received PORT to the mediastinum $(n=11)$. 
(84.6\% vs. 55.6\%) than those who also received radiotherapy to the mediastinum ( $n=11$ ); in particular, a higher number of distant metastases occurred in patients who received radiotherapy only to the costal pleura (72.7\% vs. 18.2\%).

\section{Patterns of failure}

Cancer recurred and/or metastasized during follow-up in 39 patients (38.2\%) (Fig. 5). Local recurrence, regional recurrence, and distant metastasis were found in 12 cases (11.8\%), 11 cases (10.8\%), and 25 cases (24.5\%), respectively. The sites of distant metastasis were the brain in 10 patients (9.8\%), the contralateral lung in nine patients (8.8\%), and bone in eight patients (7.8\%).

\section{Discussion and Conclusion}

Gould et al. [5] analyzed the patterns of failure and longterm outcome for 92 patients with completely resected T3NO NSCLC. The local tumor control after resection was excellent; only three patients failed locally at 4 years. The actuarial 4-year local failure-free rate was 94\%. The present study showed treatment failure in 39 patients (overall recurrence rate $38.2 \%)$, and local recurrence occurred in 12 cases (11.8\%) (Fig. 5) [5]. The 4-year actuarial regional failure-free and distant failure-free survivals were $74 \%$ and $68 \%$, respectively. Although the current study showed a higher local recurrence rate than Gould's data [5], the overall recurrence rate was

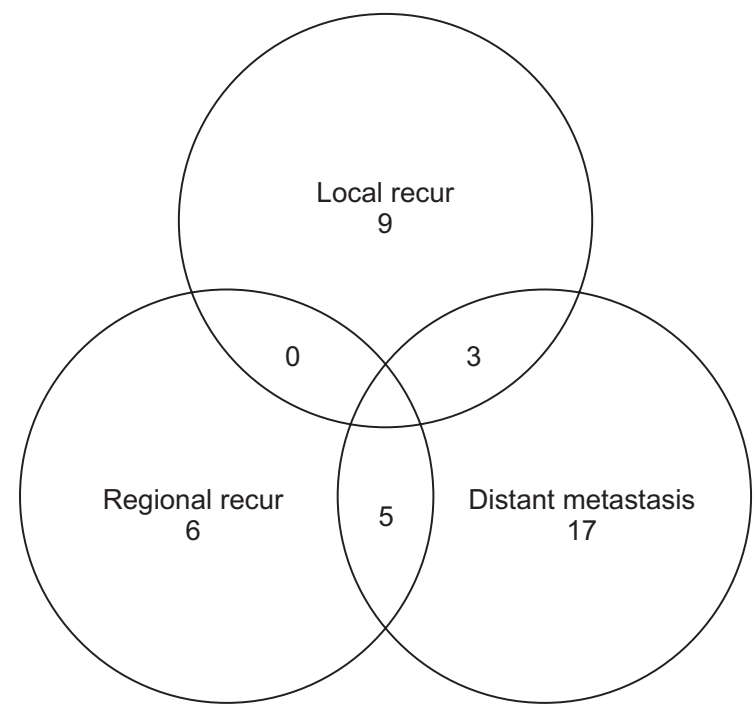

Fig. 5. Patterns of failure in resected non-small cell lung cancer T3N0 patients (number of patients). similar, and the distant failure-free survival rate was slightly lower. The fact that 5 patients with incomplete resection were included for this evaluation might influence the results.

For T3NO NSCLC, the pattern of recurrence may depend on the tumor location. Hayakawa et al. [16] reported that patients with primary tumors in the upper lobes or the superior segment of the lower lobes of the lung lived longer than those with primary tumors at any other site. Riquet et al. [17] reported that bronchial T3 tumors tended to have a better prognosis than T3 tumors infiltrating the mediastinal pleura. The present study showed a significantly lower survival rate than other subtypes in stage T3NO patients with pleural invasion (OS, $p=0.023$; DFS, $p=0.049$ ) in univariate analysis. Satoh et al. [18] previously dealt with the influence of pleural invasion on the prognosis of patients with lung cancer. They held the view that pleural invasion makes cancer cells proliferate along the pleural lymphatic flow; as a result prognosis of the patients with pleural invasion might be different from the patients with other T3 subtypes. In contrast, endobronchial tumors within $2 \mathrm{~cm}$ of the carina demonstrated a favorable prognosis compared with other T3 subtypes (OS, p $=0.003$; DFS, $p=0.006$ ).

Several studies have reported that postoperative systemic chemotherapy significantly increases the survival rate in patients with completely resected NSCLC $[1,2]$. However, the role of adjuvant chemotherapy remains under controversy for patients with completely resected T3 NSCLC without nodal involvement (T3N0). Lee et al. [19] retrospectively reviewed patients with surgically resected NSCLC invading the chest wall. They analyzed 107 patients who underwent surgical resection for NSCLC with chest wall invasion. They suggested that completion of chemotherapy is the only prognostic factor for long-term survival in 64 patients (60\%) with completely resected T3NO NSCLC infiltrating the chest wall [19]. However, no consistent institutional protocol for adjuvant chemotherapy existed in our study. As a result, adjuvant systemic chemotherapy did not show any significant increase in the survival rate in the present study.

The role of adjuvant radiotherapy in T3 NSCLC patients with chest wall invasion is still controversial [20-22]. Facciolo et al. [23] reported that the overall 5-year estimated survival for patients who underwent radiation therapy was 74.1\%, whereas in patients who did not undergo radiation therapy it was 46.7\% ( $p=0.023$ ). Gould et al. [5] reported a significant improvement in locoregional control $(p=0.05)$ for the use of adjuvant radiotherapy in patients with completely resected 
T3NO NSCLC. They showed that the 2- and 4-year regional control was $100 \%$ with radiation therapy compared with $72 \%$ and 68\%, respectively, for patients who did not receive thoracic radiation therapy. Conversely, Doddli et al. [6] assessed prognostic factors in patients with completely resected pT3 NSCLC infiltrating the chest wall and suggested that adjuvant radiotherapy does not seem to be necessary in NO patients when a complete $\mathrm{R} 0$ resection was achieved. Owing to the fact that the patients selected for adjuvant radiotherapy tended to be at risk of relapse, this retrospective study might have difficulty in showing a survival advantage for radiotherapy. On the other hand, it has been argued that the lower survival rate of the patients treated with adjuvant radiotherapy might be related to the application of inappropriate radiation dose and field $[4,5]$.

According to the present study, adjuvant radiotherapy did not cause an improvement in survival for most patients with resected T3NO NSCLC. The radiation-induced pulmonary or cardiac toxicities might indirectly affect the survival outcome. That is, the poor survival for the patients with adjuvant radiotherapy might be associated with the inadequate irradiated lung volume and the radiation ports design. In contrast, mediastinal postoperative irradiation showed a possible survival benefit in the subgroup analysis of patients with mediastinal pleura invasion. The tumor extending to the mediastinal pleura has a higher possibility to microscopically metastasize to the mediastinal lymph nodes. Therefore, mediastinal radiotherapy can be effective in the patient subgroup with mediastinal pleural invasion. In other words, adjuvant radiotherapy to the mediastinal lymph node area may be considered to improve survival in the patients with tumors involving the mediastinal pleura.

As for the limitations, the patient population of this study tended to be heterogeneous. First, it was designed as a multicenter trial, and variations of treatment among the centers might have affected the results. Second, patient enrollment was performed within a relatively long period of time (19902009). Technological improvement of radiotherapy (e.g., from 2-dimensional radiotherapy to 3-dimensional radiotherapy or intensity-modulated radiotherapy) during this period could decrease the rate of radiation toxicity and it may affect the survival rate. In addition, this study did not deal with the relationship between the treatment volume of radiotherapy and radiation toxicity. In the subgroup evaluation of the patients who had accompanying costal pleura invasion and received irradiation, the higher survival rate of the patients who received treatment only to the costal pleura compared to those who also received treatment to the mediastinum might be related to the increased radiation toxicity due to the enlarged lung irradiation volume. Furthermore, local control could be a better end-point than survival to evaluate the effect of adjuvant radiotherapy. Last but not least, this study is limited by its retrospective design. In particular, the univariate subgroup analysis result that adjuvant radiotherapy may improve survival in cases of mediastinal pleura invasion, should be evaluated in further studies owing to the relatively small sample size.

In postoperative T3NOMO patients, the tumor size was an independent prognostic factor. The subtype that showed the most favorable prognosis was the one with endobronchial tumors within $2 \mathrm{~cm}$ of the carina, which had a smaller tumor size than other subgroups. When administered after surgery, adjuvant radiotherapy is not recommended for most patients with T3NO NSCLC.

\section{Conflict of Interest}

No potential conflict of interest relevant to this article was reported.

\section{Acknowledgments}

The results of this study were presented at the 31th European Society for Radiotherapy and Oncology (ESTRO), Barcelona, Spain, May 9-13, 2012. This work was supported by a faculty research grant of Yonsei University College of Medicine for 2011 (Grant No. 6-2011-0127) and the National Research Foundation of Korea (NRF) grant funded by the Korea government (MEST) (No. 2011-0005633). The authors thank D. S. Jang for his excellent support with medical illustration.

\section{References}

1. Douillard JY, Rosell $R$, De Lena $M$, et al. Adjuvant vinorelbine plus cisplatin versus observation in patients with completely resected stage IB-IIIA non-small-cell lung cancer (Adjuvant Navelbine International Trialist Association [ANITA]): a randomised controlled trial. Lancet Oncol 2006;7:719-27.

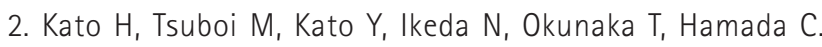
Postoperative adjuvant therapy for completely resected earlystage non-small cell lung cancer. Int J Clin Oncol 2005;10:15764. 
3. Lally BE, Zelterman D, Colasanto JM, Haffty BG, Detterbeck FC, Wilson LD. Postoperative radiotherapy for stage II or III nonsmall-cell lung cancer using the surveillance, epidemiology, and end results database. J Clin Oncol 2006;24:2998-3006.

4. Park YJ, Ahn YC, Lim DH, et al. Postoperative radiation therapy for chest wall invading pT3NO non-small cell lung cancer: elective lymphatic irradiation may not be necessary. J Korean Soc Ther Radiol Oncol 2003;21:253-60.

5. Gould PM, Bonner JA, Sawyer TE, Deschamps C, Lange CM, $\mathrm{Li} \mathrm{H}$. Patterns of failure and overall survival in patients with completely resected T3 NO MO non-small cell lung cancer. Int J Radiat Oncol Biol Phys 1999;45:91-5.

6. Doddoli C, D'Journo B, Le Pimpec-Barthes F, et al. Lung cancer invading the chest wall: a plea for en-bloc resection but the need for new treatment strategies. Ann Thorac Surg 2005;80: 2032-40.

7. Rami-Porta R, Ball D, Crowley J, et al. The IASLC Lung Cancer Staging Project: proposals for the revision of the T descriptors in the forthcoming (seventh) edition of the TNM classification for lung cancer. J Thorac Oncol 2007;2:593-602.

8. Rena O, Massera F, Robustellini M, et al. Use of the proposals of the international association for the study of lung cancer in the forthcoming edition of lung cancer staging system to predict long-term prognosis of operated patients. Cancer J 2010;16:176-81.

9. Van Schil PE, Hendriks JM, Hertoghs $M$, Lauwers $P$, Choong C. Current surgical treatment of non-small-cell lung cancer. Expert Rev Anticancer Ther 2011;11:1577-85.

10. Dilege $S$, Toker A, Tanju S, et al. Chest wall invasion in lung cancer patients. Acta Chir Belg 2003;103:396-400.

11. Lally BE, Detterbeck FC, Geiger AM, et al. The risk of death from heart disease in patients with nonsmall cell lung cancer who receive postoperative radiotherapy: analysis of the Surveillance, Epidemiology, and End Results database. Cancer 2007;110:911-7.

12. Yoon $\mathrm{HI}$, Kim YB, Lee CG, et al. Division of the $\mathrm{N} 2$ stage according to the multiplicity of the involved nodal stations may be necessary in the N2-NSCLC patients who are treated with postoperative radiotherapy. J Korean Soc Ther Radiol
Oncol 2009;27:126-32.

13. Bogart JA, Aronowitz JN. Localized non-small cell lung cancer: adjuvant radiotherapy in the era of effective systemic therapy. Clin Cancer Res 2005;11(13 Pt 2):5004s-5010s.

14. Voltolini L, Rapicetta C, Luzzi L, et al. Lung cancer with chest wall involvement: predictive factors of long-term survival after surgical resection. Lung Cancer 2006;52:359-64.

15. Bonner JA, Spencer SA. Postoperative radiotherapy in nonsmall-cell lung cancer warrants further exploration in the era of adjuvant chemotherapy and conformal radiotherapy. J Clin Oncol 2006;24:2978-80.

16. Hayakawa K, Mitsuhashi N, Saito $Y$, et al. Impact of tumor extent and location on treatment outcome in patients with stage III non-small cell lung cancer treated with radiation therapy. Jpn J Clin Oncol 1996;26:221-8.

17. Riquet $M$, Lang-Lazdunski $L$, Le $P B$, et al. Characteristics and prognosis of resected T3 non-small cell lung cancer. Ann Thorac Surg 2002;73:253-8.

18. Satoh $Y$, Ishikawa $Y$, Inamura $K$, Okumura $S$, Nakagawa $K_{\text {, }}$ Tsuchiya E. Classification of parietal pleural invasion at adhesion sites with surgical specimens of lung cancer and implications for prognosis. Virchows Arch 2005;447:984-9.

19. Lee $C Y$, Byun CS, Lee JG, et al. The prognostic factors of resected non-small cell lung cancer with chest wall invasion. World J Surg Oncol 2012;10:9.

20. Patterson GA, Ilves R, Ginsberg RJ, Cooper JD, Todd TR, Pearson FG. The value of adjuvant radiotherapy in pulmonary and chest wall resection for bronchogenic carcinoma. Ann Thorac Surg 1982;34:692-7.

21. Burkhart HM, Allen MS, Nichols FC 3rd, et al. Results of en bloc resection for bronchogenic carcinoma with chest wall invasion. J Thorac Cardiovasc Surg 2002;123:670-5.

22. Allen MS, Mathisen DJ, Grillo HC, Wain JC, Moncure AC, Hilgenberg AD. Bronchogenic carcinoma with chest wall invasion. Ann Thorac Surg 1991;51:948-51.

23. Facciolo F, Cardillo G, Lopergolo M, Pallone G, Sera F, Martelli $M$. Chest wall invasion in non-small cell lung carcinoma: a rationale for en bloc resection. J Thorac Cardiovasc Surg 2001; 121:649-56. 